\title{
Periodontal Status (CPITN) of Six-to \\ Fifteen-Year-Old West Malaysian Aborigines (Proto-Malays)
}

\author{
Rahimah Abdul-Kadir, DDS(Indon), MSD(Indiana), \\ MPH(Michigan) and Ahmad Tajuddin Yassin, BDS(S'pore), \\ DDPHRCS(England)
}

(Received 9 October and accepted 16 October 1989)

Key words: aborigines, semi-primitive, developing country, CPITN

\begin{abstract}
A survey of 303 six- to fifteen-year-old Proto-Malay children was carried out in Selangor, West Malaysia. The examinations were carried out using a chair-table and natural daylight. Periodontal status was assessed using the Community Periodontal Index of Treatment Needs (CPINT). The findings revealed a generally low prevalence of periodontal disease affecting only $25.08 \%$ of the sample examined. Of these, $15.72 \%$ were found to have a score of 1 (bleeding of the gingiva), while $9.36 \%$ had calculus on the tooth surface. In terms of treatment needs, $24.75 \%$ required dental health education. The relationship between these findings and the possible causes are discussed.
\end{abstract}

\section{Introduction}

The aborigines of West Malaysia, officially known as the "Orang Asli" comprise $1 \%$ of the total West Malaysian population ${ }^{[1]}$. As of 1986 there were 68,935 Orang Asli in West Malaysia ${ }^{[2]}$. They are made up of three major ethnic groups, namely the Negritos, Senois and Proto-Malays. The Negritos, small, dark skinned with wooly hair and leading a nomadic life, live in the northern part of West Malaysia. The Senois are thought to be related to a variety of hill people found in various parts of South East Asia. The Proto-Malays, who lead a more settled lifestyle, are found in the southern part of the Malay peninsula ${ }^{[1,3,4]}$.

In the state of Selangor, the Proto-Malays form the major ethnic group. The population according to the 1986 census was 8,388 , of which $19.11 \%(1,114)$ were children $^{[2]}$. They generally live in isolated villages located in the hilly areas of the state. The inhabitants of each village are usually small in number (not more than 150 persons). By natural selection, once a community grows, it automatically splits $^{[1,3]}$.

Lecturers, Department of Preventive Dentistry, Faculty of Dentistry, University of Malaya. Correspondence to: Dr Rahimah Abdul-Kadir, Department of Preventive Dentistry, Faculty of Dentistry, University of Malaya, 59100 Kuala Lumpur, MALA YSIA. TEL: 03-7567311 ext 264. This project was supported financially by a Vote $F$ grant from the University of Malaya, Kuala Lumpur, MALAYSIA. 
As far as can be determined, the studies that have been done relate mostly to their lifestyle, culture and religious beliefs ${ }^{[5,6]}$, and few studies on their medical condition have been reported ${ }^{[7,8]}$. However, no formal report has been published concerning their dental health.

Recognizing this lack of information, a preliminary survey on a group of schoolchildren in a small community of Proto-Malays of the Temuan tribe in Selangor was done in mid $1987^{[9]}$. The findings from the survey indicated that the prevalence of periodontal disease was low. The findings were, however, limited to a small sample size, and therefore it is suggested that a more representative sample is necessary to validate them.

The objectives of the survey were: (1) to determine the periodontal status of 6 to 15-year-old Selangor Proto-Malay children, (2) to provide data for use in estimating needs for periodontal treatment, and (3) to provide an initial exposure of the community to dental treatment in an effort to reduce fear and anxiety of such treatment. For many of the population, this was the first time they had received a dental check.

\section{Materials and Methods}

In the preliminary survey at Bukit Lanjan, Selangor, in mid $1987^{[9,10]}$, the factors that influenced the children's maximum participation were shyness, fear and anxiety. Another was the small total enrollment at the schools and the high absentee rate of at least $60 \%$ daily for reasonably long periods. Recognizing these problems, and because the villages were only accessible after several hours' driving time, it was decided that for the purpose of this study and assuming that the villages did not differ significantly from one another with respect to lifestyle and culture, only Orang Asli schools with maximum enrollment were selected. In addition, every child registered at the school was included in the sample. As an added strategy, only the headmasters of the schools were informed of the date and time of the survey team's visit in advance. In spite of all efforts to maximize the participation in the present study, only 303 of the 569 Proto-Malay children (registered in 1988) were available on the day of examination. As far as could be determined, all of these children were of pure aboriginal parentage.

A cross-sectional picture of the periodontal status of the Orang Asli children was assessed using the Community Periodontal Index for Treatment Needs (CPITN), utilizing six index teeth (teeth $16,11,26,36,31,46$ ) to represent the six sextants of the mouth as recommended for populations under 20 years of age ${ }^{[11-12]}$. Examination was carried out using a chair-table and natural daylight. A special probe (WHO 621) as described by EMSLIE $^{[13]}$ was used in the examination.

All observations collected in this survey were recorded directly on the survey form. Data analysis was done on an IBM PC compatible microcomputer and analyzed using appropriate statistical software.

\section{Results}

The distribution of the survey sample was as shown in Table 1. Of the 303 
Table 1 Distribution of the survey sample by age and sex

\begin{tabular}{crrrrrr}
\hline $\begin{array}{c}\text { Age } \\
\text { (years) }\end{array}$ & $\mathrm{N}$ & \multicolumn{1}{c}{ Male } & \multicolumn{2}{c}{ Female } & \multicolumn{2}{c}{ Total } \\
\hline 6 & 9 & 60.00 & 6 & 40.00 & 15 & 4.92 \\
7 & 16 & 51.61 & 15 & 48.39 & 31 & 10.62 \\
8 & 27 & 50.94 & 26 & 49.06 & 53 & 17.38 \\
9 & 27 & 46.55 & 31 & 53.45 & 58 & 10.02 \\
10 & 23 & 67.65 & 11 & 32.35 & 34 & 11.15 \\
11 & 19 & 50.00 & 19 & 50.00 & 38 & 12.46 \\
12 & 14 & 36.84 & 24 & 63.16 & 38 & 12.46 \\
13 & 10 & 45.45 & 12 & 54.55 & 22 & 7.21 \\
14 & 4 & 57.14 & 3 & 42.86 & 7 & 2.39 \\
15 & 3 & 42.86 & 4 & 57.14 & 7 & 2.39 \\
\hline $6-15$ & 152 & 50.17 & 151 & 49.33 & 303 & 100.00 \\
\hline
\end{tabular}

children examined, $50.17 \%$ were males and $49.33 \%$ were females. The ratio of male children examined to female children for various ages was $1: 1$ with the exception of 10- and 12-year-olds, for whom the ratios were $2: 1$ and $1: 2$, respectively.

The periodontal status of the 303 children examined is shown in Table 2. Of the total examined, 299 were dentate and considered in the final analysis. The findings showed that $74.92 \%$ of these children had healthy gingivae (score 0 ). In

Table 2 Periodontal status (CPITN) of 6- to 15-year-old Orang Asli children in Selangor, West Malaysia

\begin{tabular}{|c|c|c|c|c|c|c|c|c|}
\hline \multirow{2}{*}{$\begin{array}{c}\text { Age } \\
\text { (years) }\end{array}$} & \multicolumn{2}{|c|}{ Number } & \multicolumn{2}{|c|}{ Score 0} & \multicolumn{2}{|c|}{ Score 1} & \multicolumn{2}{|c|}{ Score 2} \\
\hline & Examined & Dentate & $\mathrm{X}$ & $\%$ & $X$ & $\%$ & $X$ & $\%$ \\
\hline 6 & 15 & 13 & 5.69 & 76.92 & 0.23 & 23.08 & 0.00 & 0.00 \\
\hline 7 & 31 & 29 & 4.93 & 79.31 & 0.38 & 10.34 & 0.21 & 10.34 \\
\hline 8 & 53 & 53 & 5.45 & 81.13 & 0.25 & 13.21 & 0.08 & 5.66 \\
\hline 9 & 58 & 58 & 5.55 & 74.14 & 0.45 & 20.69 & 0.07 & 5.17 \\
\hline 10 & 34 & 34 & 5.62 & 76.47 & 0.35 & 17.65 & 0.09 & 5.88 \\
\hline 11 & 38 & 38 & 5.53 & 76.32 & 0.47 & 13.16 & 0.16 & 10.53 \\
\hline 12 & 38 & 38 & 5.39 & 68.42 & 0.61 & 15.79 & 0.21 & 15.79 \\
\hline 13 & 22 & 22 & 5.04 & 63.64 & 0.91 & 4.55 & 0.86 & 31.82 \\
\hline 14 & 7 & 7 & 5.57 & 71.43 & 0.43 & 28.57 & 0.00 & 0.00 \\
\hline 15 & 7 & 7 & 5.71 & 85.71 & 0.29 & 14.29 & 0.00 & 0.00 \\
\hline $6-15$ & 303 & 299 & 5.44 & 74.92 & 0.41 & 15. 27 & 0.15 & 9.36 \\
\hline
\end{tabular}

$\mathrm{X}$ : Mean number of sextants/child with CPITN scores $0,1,2$

$\%$ : Percentage distribution of subjects recording the maximum CPITN score of $0,1,2$

addition, $15.72 \%$ were found to have a score of 1 , denoting bleeding of the gingiva, while $9.36 \%$ had calculus formation on their tooth surface (score 2). On average, at least five sextants of the gingiva were healthy. The mean number of bleeding sextants was 0.42 , while only 0.15 sextant per child affected had calculus on the tooth surface.

Sex-specific comparison revealed a generally low prevalence of bleeding gingiva (score 1) in females between 7-9 years old as compared with their male peers (Fig. 1). However, the reverse prevalence was observed in females 10-14 years 

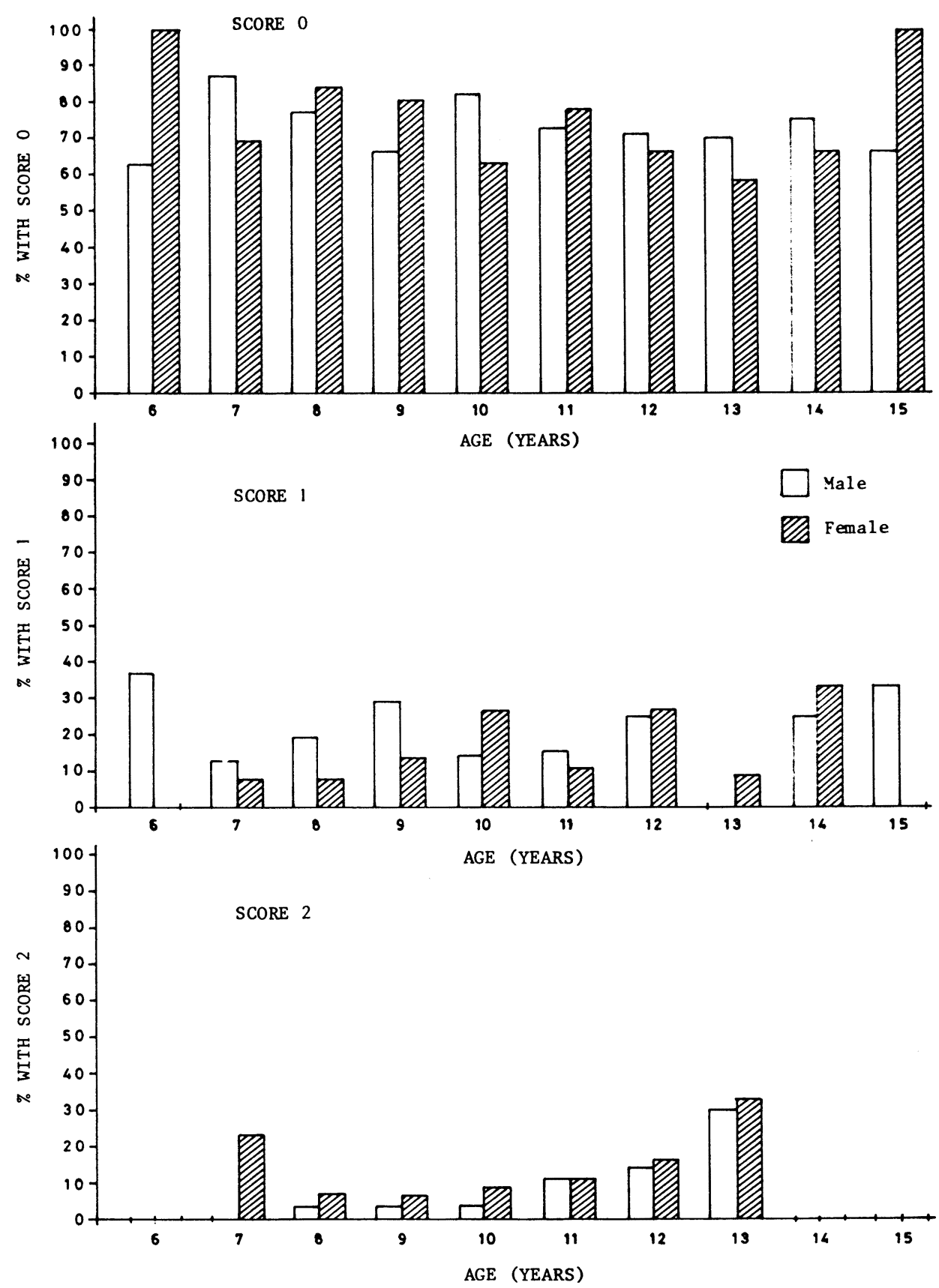

Fig. 1. Periodontal status (CPITN) of the aborigine children by sex 
old. In terms of calculus formation on the tooth surface (score 2), the prevalence was higher in females 7-10 years old. Beyond that age, the prevalence appeared to be similar in both sexes.

As for periodontal treatment needs in this sample, $24.75 \%$ of children appeared to require oral hygiene education (OHE), while $9.36 \%$ required scaling (Table 3 ). While the percentage of children requiring OHE was generally constant for all age

Table 3 Periodontal treatment needs of 6- to 15-year-old Orang Asli children

\begin{tabular}{|c|c|c|c|c|c|}
\hline \multirow{2}{*}{$\begin{array}{c}\text { Age } \\
\text { (years) }\end{array}$} & \multicolumn{2}{|c|}{ Number } & \multirow{2}{*}{$\begin{array}{c}\text { OHE } \\
\%\end{array}$} & \multicolumn{2}{|c|}{ Scaling } \\
\hline & Examined & Dentate & & $\%$ & $\mathrm{X}$ \\
\hline 6 & 15 & 13 & 23.08 & 0.00 & 0.00 \\
\hline 7 & 31 & 29 & 20.69 & 10.34 & 0.21 \\
\hline 8 & 53 & 53 & 18.87 & 5.66 & 0.08 \\
\hline 9 & 58 & 58 & 25.86 & 5.17 & 0.07 \\
\hline 10 & 34 & 34 & 23.53 & 5.88 & 0.09 \\
\hline 11 & 38 & 38 & 23.68 & 10.53 & 0.16 \\
\hline 12 & 38 & 38 & 31.58 & 15.79 & 0.21 \\
\hline 13 & 22 & 22 & 36.36 & 31.82 & 0.86 \\
\hline 14 & 7 & 7 & 28.57 & 0.00 & 0.00 \\
\hline 15 & 7 & 7 & 14.29 & 0.00 & 0.00 \\
\hline $6-15$ & 303 & 299 & 24.75 & 9.36 & 0.33 \\
\hline
\end{tabular}

$\mathrm{X}$ : Mean number of sextants/child requiring treatment

$\%$ : Percentage of children requiring treatment

groups, the necessity of scaling was higher among 7-year-olds, gradually decreasing and stabilizing at the age of 10 , followed by an upward trend until 13 years of age.

\section{Discussion}

The findings of this survey indicate a generally low prevalence of periodontal problems (bleeding and calculus) among Proto-Malay children in Selangor. In addition, no peridontal pockets were detected in any of the children examined. Previous data collected in line with the World Health Organization's (WHO) guidelines $^{[14]}$ on 6- to 15 -year-old schoolchildren in West Malaysia ${ }^{[15]}$, Sarawak ${ }^{[16]}$ and Sabah ${ }^{[17]}$ showed a much higher prevalence of gingival bleeding and calculus formation than those observed in this study (Table 4). Although it must be

Table 4 Comparison of periodontal prevalence in Malaysian

\begin{tabular}{|c|c|c|c|c|c|}
\hline \multirow[t]{2}{*}{ Region } & \multirow{2}{*}{$\begin{array}{c}\text { Age } \\
\text { (Years) }\end{array}$} & \multirow[t]{2}{*}{ Year } & \multicolumn{2}{|c|}{ Percentage with } & \multirow{2}{*}{$\begin{array}{c}\text { Mean gingival } \\
\text { units affected } \\
\hat{X}\end{array}$} \\
\hline & & & Calculus & $\begin{array}{c}\text { Inflamed } \\
\text { gingiva }\end{array}$ & \\
\hline West Malaysia* & $6-15$ & 1971 & 49.83 & 60.56 & 2.81 \\
\hline Sarawak* & $6-15$ & 1981 & 49.28 & 51.07 & 1.83 \\
\hline Sabah* & $6-15$ & 1985 & 54.54 & 74.68 & 3.64 \\
\hline Asli WMsia & $6-15$ & 1988 & 9.36 & 15.72 & 0.41 \\
\hline
\end{tabular}

$\widehat{\mathrm{X}}$ : Number of inflamed papilla per tooth per child

* Based on the guidelines of the World Health Organization ${ }^{[14]}$ 
recognized that because different methodologies were used it is not possible to make direct comparisons, these findings provide an overview of the periodontal status of 6- to 15-year-olds among racial groups in Malaysia.

The findings from this study also indicate that aboriginal children do not seem to attach much importance to dental hygiene. This is evidenced by the fact that at least $24.75 \%$ of those affected required dental health instruction. In addition, more than $70 \%$ of children examined had extrinsic stains on their tooth surface. This observation seems to be closely related to the findings of a previous preliminary study ${ }^{[10]}$ in which $84 \%$ of the children examined admitted that they never used toothbrushes provided by the Malaysian Ministry of Education.

In the light of other unknown environmental and cultural factors related to dental habits, it must be emphasized that the findings of this survey should be viewed with caution. In spite of the observed presence of a generally poor attitude and lack of motivation to practice good oral hygiene, the low prevalence of periodontal disease observed in this study can probably be partially attributed to the more fibrous and semi-primitive diet that the aborigines have chosen to retain $^{[8,18]}$, which may have a self-cleansing effect. As a substitute for toothbrushes, the use of charcoal and sand may also have contributed to this low prevalence ${ }^{[8]}$.

The present findings also suggest that curative treatment is not an immediate requirement. Instead, as a prevention strategy, dental health education seems to be of priority and should be provided on a more systematic and frequent basis. Studies ${ }^{[19,20]}$ have shown that in the absence of adequate oral hygiene, periodontal disease may progress with age. Prevention efforts should also therefore be extended to all age groups. It is recognized that the existing communication gap and cultural differences are problems which can influence the acceptance of new changes by the community. These problems can be partly overcome if a primary health care approach, i.e. selection and training of the aborigines themselves to perform dental health education, is introduced.

This study has also provided an insight for future investigations in this community. The sampling problem appears to be universal in aboriginal studies $^{[8,9,21]}$. Apart from fear and anxiety, the culture and environment play an important role in the everyday life of the Orang Asli. The school attendance of the children in this study was dependent on these factors. This was especially apparent on one occasion prior to the team's visit, when the level of the river in one of the villages rose after two days of heavy rain, and most of the children spent their time playing in the river, so that school attendance dropped by $60 \%$. Thus school is not considered important. The existing problems of accessibility, general attitude and communication in this population should be considered by future investigators at the planning stage.

\section{Acknowledgements}

The investigators extend their appreciation to the University of Malaya, the Department of Orang Asli Affairs (JHEOA), and the Dental Division Ministry of Health for their financial and moral support. 


\section{References}

[1] ImIN, I.: A brief note on the Orang Asli of Peninsular Malaysia and their administration, Department of Orang Asli Affairs: Kuala Lumpur, 1981

[2] Department of Orang Asli Affairs (JHEOA). 1986 Orang Asli Census. JHEOA: Kuala Lumpur, Malaysia, 1986 (unpublished document)

[3] Williams-Hunt, P. D. R.: An introduction to the Malayan aborigines, Malayan Govt Press, Kuala Lumpur, 1952

[4] Jones, A.: The Orang Asli: an outline of their progress in modern Malaya, JSAH., 9, 286-305, 1968

[5] Evans, I. H. N.: Beliefs, customs and folk tales of the Behrag Valley Senoi, JFMS Mus., 7, $143-262,1918$

[6] CAREY, I.: The religion problem among the Orang Asli, JMBRAS, 43, 155-160, 1970

[7] Bolton, J. M.: Medical services to the aborigines in West Malaysia, Brit Med J., 2, 818-823, 1968

[8] Soong, F. S.: Some beliefs and practices affecting the health of the aborigines (Orang Asli) of Bukit Lanjan, West Malaysia, SEA J Trop Med and Public Health, 3, 267-275, 1972

[9] ABDUL-K ADIR, R.: Dental health status of Temuan tribe schoolchildren of West Malaysia, $\boldsymbol{J}$ Dent Res., 67, 792 (abstract), 1988

[10] Abdul-Kadir, R. and Adnan, N. Md.: Prevalence of dental caries in a selected group of West Malaysian Orang Asli, Tropical Dent J., 12, 7-12, 1989

[11] Ainamo, J., Parviainen, K. and Murtomaa, H.: Reliability of the needs at 13-15 years of age, Int Dent J., 34, 214-216, 1984

[12] Ainamo, J., Nordblad, A and Kallio, P.: Use of the CPITN in populations under 20 years of age, lnt Dent J., 34, 285-287, 1984

[13] Emsuie, R. D.: The 621 periodontal probe, lnt Dent $J$ 30: 287, 1980

[14] World Health Organization. Oral health surveys: Basic methods, 1st edition, Oral Health Unit, WHO, Geneva, 1971

[15] Dental Division. Dental epidemiological survey of schoolchildren in West Malavsia, August 1970-May 1971, Kuala Lumpur, Ministry of Health, 1972

[16] Dental Division. Dental epidemiological survey of schoolchildren in Sarawak, Kuala Lumpur, Ministry of Health, 1983

[17] Dental Division. Dental epidemiological survey of schoolchildren in Sabah, Kuala Lumpur, Ministry of Health, 1985

[18] Robson P, Bolton JM, Dugdale AE.: The nutrition of the Malaysian aboriginal children, Am J Clinical Nutr., 26, 95-100, 1973

[19] Haffajee, A. D., Socransky, S. S., Goodson, J. M.: Periodontal disease activity, J Periodontal Res., 17, 521-522, 1982

[20] Socransky, S. S., Haffajee, A. D., Goodson, J. M. et al.: New concepts of destructive periodontal disease, J Clin Periodontol., 11, 21-31, 1984

[21] Chen, J. A.: Dental status of the Penans along the Melinau and Terawan Rivers, Baram, Sarawak, Med J Msia., 41, 225-229, 1986 